\title{
ISAR-LEFT MAIN
}

\section{A Randomized Clinical Trial on Drug-Eluting Stents for Unprotected Left Main Lesions}

\section{Eine randomisierte klinische Studie zum Einsatz von Medikamente freisetzenden Stents bei ungeschützter Hauptstammstenose}

Hintergrund und Problemstellung: Der Stellenwert der elektiven interventionellen Behandlung von Patienten mit ungeschützter Hauptstammstenose ist umstritten, da bisher lediglich Registeranalysen vorliegen und Daten zu den Langzeitergebnissen fehlen. Für Notfallpatienten existieren inzwischen Registerdaten, die für diese Patientengruppe eine akzetable

\begin{tabular}{ll}
\hline Methodik & \\
\hline Studiendesign & $\begin{array}{l}\text { Randomisierte, nichtverblindete, kontrollierte } \\
\text { Nichtunterlegenheitsstudie (Taxus gegenüber } \\
\text { Cypher nicht unterlegen) } \\
\text { Kombinierter Endpunkt aus Tod, Herzinfarkt } \\
\text { oder "target lesion revascularization“ (TLR) } \\
\text { nach 12 Monaten }\end{array}$ \\
Sekundäre Endpunkte & $\begin{array}{l}\text { Tod, Herzinfarkt, Apoplex, Stentthrombose, } \\
\text { 2-Jahres-MACE, 2-Jahres-Mortalität } \\
\text { Einschlusskriterien: }\end{array}$ \\
Patientenauswahl & $-\geq 50 \%$ Hauptstammstenose (Gefäßdiameter \\
& $\leq 4,5$ mmm) + \\
& $\bullet$ symptomatische Ischämie und/oder \\
& $\begin{array}{l}\text { Ausschlusskriterien: } \\
\text { Kardiogener Schock, ST-Hebungsinfarkt, } \\
\text { In-Stent-Restenose, Z.n. Bypassoperation, } \\
\text { "staged procedure“ }\end{array}$ \\
& 2
\end{tabular}

\begin{tabular}{lcc}
\hline Ergebnisse $\left({ }^{*} \mathrm{p}<0,05\right)$ & & \\
\hline & Taxus-Stent & Cypher-Stent \\
Patientenanzahl & $\mathrm{n}=302$ & $\mathrm{n}=305$ \\
Patientencharakteristika & & \\
Alter & $68,8 \pm 10,3$ & $69,3 \pm 9,3$ \\
Diabetes & $30 \%$ & $28 \%$ \\
EuroSCORE & $4,7 \pm 3,5$ & $4,4 \pm 3,2$ \\
Distale Läsion & $63 \%$ & $63 \%$ \\
Trifurkation & $25 \%$ & $24 \%$ \\
Stenttechnik & & \\
Culotte/T/einfach & $49 \% / 2 \% / 49 \%$ & $48 \% / 1 \% / 51 \%$ \\
Primärer Endpunkt & $13,6 \%$ & $15,8 \%$ \\
Tod & $5,0 \%$ & $6,6 \%$ \\
Herzinfarkt & $5,0 \%$ & $4,6 \%$ \\
Schlaganfall & $1,7 \%$ & $1,0 \%$ \\
Tod/MI/Schlaganfall & $9,6 \%$ & $10,2 \%$ \\
Angiographische Restenose & $16,0 \%$ & $19,4 \%$ \\
2-Jahres-TLR & $6,5 \%$ & $7,8 \%$ \\
Stentthrombose (2 Jahre) & $0,3 \% / 0,0 \%$ & $0,7 \% / 0,3 \%$ \\
definitiv/wahrscheinlich & & \\
& &
\end{tabular}

Schlussfolgerung und Kommentar: Die Hypothese der Studie konnte bestätigt werden: Der Einsatz des Taxus-Stents ist dem Cypher-Stent bei Patienten mit ungeschützter Hauptstammstenose nicht unterlegen. In Ergänzung zur SYNTAX-Studie
Evidenzgrundlage schaffen. Nachdem die SYNTAX-Studie als erste randomisierte Studie den Vergleich Bypassoperation versus einen Medikamente freisetzenden Stent (DES, Taxus) lieferte, hatte ISAR LEFT-MAIN zum Ziel, Taxus- und Cypher-Stents bei dieser Indikation zu vergleichen. Studienleiter war A. Kastrati, München.

Evidence-based-Medicine-(EBM-)Score

\begin{tabular}{|c|c|c|}
\hline Klinischer primärer Endpunkt & $\begin{array}{l}\text { Ja }=3 \\
\text { Nein }=0\end{array}$ & 3 \\
\hline Doppelblind (einschließlich Ärzten) & $\begin{array}{l}\mathrm{Ja}=1 \\
\mathrm{Nein}=0\end{array}$ & 0 \\
\hline $\begin{array}{l}\text { Beobachtungsintervall für den primären Endpunkt } \\
\geq 6 \text { Monate }\end{array}$ & $\begin{array}{l}\mathrm{Ja}=1 \\
\text { Nein }=0\end{array}$ & 1 \\
\hline Multicenter (mindestens 3 Zentren) & $\begin{array}{l}\text { Ja }=1 \\
\text { Nein }=0\end{array}$ & 0 \\
\hline $\begin{array}{l}\text { Externes und vom Steering Committee unabhängiges } \\
\text { Clinical Event Committee/DSMB (Datensicherheit- } \\
\text { Monitoring-Board) }\end{array}$ & $\begin{array}{l}\text { Ja }=1 \\
\text { Nein }=0\end{array}$ & 0 \\
\hline Primärer Endpunkt erreicht & $\begin{array}{l}\mathrm{Ja}=1 \\
\text { Nein }=0\end{array}$ & 1 \\
\hline Power von $\geq 80 \%$ für den primären Endpunkt erreicht & $\begin{array}{l}\text { Ja }=1 \\
\text { Nein }=0\end{array}$ & 1 \\
\hline $\begin{array}{l}\text { Anteil des Follow-up der Patienten für } \\
\text { angiographischen primären Endpunkt } \geq 80 \% \text { und } \\
\geq 95 \% \text { für klinischen primären Endpunkt }\end{array}$ & $\begin{array}{l}\mathrm{Ja}=1 \\
\text { Nein }=0\end{array}$ & 1 \\
\hline Gesamt-EBM-Score & & \\
\hline
\end{tabular}

"Silber-Score" für randomisierte, kontrollierte Studien (RaCT) oder kontrollierte Registerstudien (ReCT mit präspezifizierter Kontrollgruppe und Power-Kalkulation); es sind maximal 10 Scorepunkte möglich (J Interv Cardiol 2006;19:485-92)

(Herz 2008;33:535) kann zudem die Schlussfolgerung gezogen werden, dass die PCI bei dieser Indikation sicher und machbar ist. Die vorliegenden Daten aus ISAR LEFT-MAIN lassen auch nach 2 Jahren keinen Unterschied der beiden Stents hinsichtlich Mortalität oder MACE erkennen. Unklar bleibt bisher die beste PCI-Technik: Aus ISAR LEFT-MAIN liegt bisher noch keine Subgruppenanalyse vor, die die Ergebnisse der Culotte-Technik mit dem einfachen Stenting vergleicht. Die Datenlage für den Einsatz des Taxus-Stents in diesem Bereich ist durch die SYNTAX-Studie sehr gut geworden; die ISAR-LEFT-MAIN-Studie zeigt nun vergleichbare Ergebnisse für den Cypher-Stent. Auch war die Rate an Stentthrombosen relativ niedrig. Ob dies mit der Dosierung (Clopidogrel $2 \times 75 \mathrm{mg}$ bis zur Entlassung, dann $1 \times 75 \mathrm{mg}$ unbegrenzt und 200 mg ASS) oder mit dem größeren Gefäßdurchmesser bzw. besseren Fluss im Hauptstammbereich zusammenhängt, bleibt zu diskutieren.

M.W. Bergmann, Hamburg 